\title{
Epigenetic changes induced by adenosine augmentation therapy prevent epileptogenesis
}

\author{
Rebecca L. Williams-Karnesky, ${ }^{1,2}$ Ursula S. Sandau, ${ }^{1}$ Theresa A. Lusardi, ${ }^{1}$ Nikki K. Lytle, ${ }^{1}$ \\ Joseph M. Farrell,, Eleanor M. Pritchard,, ${ }^{3}$ David L. Kaplan, ${ }^{3}$ and Detlev Boison ${ }^{1,2}$
}

${ }^{1}$ RS Dow Neurobiology Laboratories, Legacy Research Institute, Portland, Oregon, USA. 2Department of Neurology, Oregon Health and Sciences University, Portland, Oregon, USA. ${ }^{3}$ Department of Biomedical Engineering, Tufts University, Medford, Massachusetts, USA.

\begin{abstract}
Epigenetic modifications, including changes in DNA methylation, lead to altered gene expression and thus may underlie epileptogenesis via induction of permanent changes in neuronal excitability. Therapies that could inhibit or reverse these changes may be highly effective in halting disease progression. Here we identify an epigenetic function of the brain's endogenous anticonvulsant adenosine, showing that this compound induces hypomethylation of DNA via biochemical interference with the transmethylation pathway. We show that inhibition of DNA methylation inhibited epileptogenesis in multiple seizure models. Using a rat model of temporal lobe epilepsy, we identified an increase in hippocampal DNA methylation, which correlates with increased DNA methyltransferase activity, disruption of adenosine homeostasis, and spontaneous recurrent seizures. Finally, we used bioengineered silk implants to deliver a defined dose of adenosine over 10 days to the brains of epileptic rats. This transient therapeutic intervention reversed the DNA hypermethylation seen in the epileptic brain, inhibited sprouting of mossy fibers in the hippocampus, and prevented the progression of epilepsy for at least 3 months. These data demonstrate that pathological changes in DNA methylation homeostasis may underlie epileptogenesis and reversal of these epigenetic changes with adenosine augmentation therapy may halt disease progression.
\end{abstract}

\section{Introduction}

Epilepsy is the third most common neurological disorder, affecting nearly 50 million people worldwide. Despite decades of research, satisfactory seizure suppression is still only achieved in just over half of affected individuals. Current antiepileptic therapies fail to address the underlying causes of epilepsy and do not halt epileptogenesis (1). Epileptogenesis is characterized by a progressive increase in frequency and severity of spontaneous recurrent seizures (SRS). Several mechanisms are thought to be implicated in the epileptogenic cascade, including neuroinflammatory responses, selective neuronal cell loss, mossy fiber sprouting, aberrant connectivity, and gliosis coupled with adenosine (ADO) dysfunction. One potential unifying factor behind many of the pathological changes in epileptogenesis may be epigenetic modifications, which are likely further potentiated by epileptogenesis itself $(2,3)$. Epigenetic modifications, which alter gene transcription without modifying the underlying DNA sequence, are highly plastic and can respond rapidly to environmental cues, an important endogenous mechanism for temporally and spatially controlling gene expression. Changes in histone acetylation and methylation as well as changes in DNA methylation, once thought to occur only in dividing cells, have been shown to also occur in mature cells in the CNS $(4,5)$. Tellingly, these changes occur regularly and rapidly. Even a single episode of neural synchronization exceeding 30 seconds in the hippocampus induces DNA methylation-dependent alterations in transcription of immediate early genes and initiates a cascade of transcription factors, contributing to long-term neuronal and circuit alterations (6).

Authorship note: Rebecca L. Williams-Karnesky, Ursula S. Sandau, and Theresa A. Lusardi contributed equally to this work.

Conflict of interest: The authors have declared that no conflict of interest exists. Citation for this article: J Clin Invest. 2013;123(8):3552-3563. doi:10.1172/JCI65636.
Methylation of DNA in the CNS has attracted increasing attention recently, with new research showing activity-induced proliferation of neural precursor cells via active DNA demethylation (5). Altered DNA methylation in the brain has also been implicated in psychiatric and neurological conditions, including epilepsy $(5,7)$. The methylation hypothesis of epileptogenesis suggests that seizures by themselves can induce epigenetic chromatin modifications and thereby aggravate the epileptogenic condition (2). Despite new insights into the role of pathological DNA methylation changes in disease and the fact that 2 DNA methyltransferase (DNMT) inhibitors are currently FDA approved (azacytidine and decitabine), direct manipulation of DNA methylation has not been tested in human epilepsy or in animal models of the disease (8).

DNA methylation requires the donation of a methyl group from S-adenosylmethionine (SAM), a process that is facilitated by DNMT enzymes (Figure 1A). The resulting product, S-adenosylhomocysteine $(\mathrm{SAH})$, is then further converted into $\mathrm{ADO}$ and homocysteine (HCY) by SAH hydrolase. Critically, the equilibrium constant of the SAH hydrolase enzyme lies in the direction of SAH formation (9); therefore, the reaction will only proceed when $\mathrm{ADO}$ and HCY are constantly removed $(9,10)$. In the adult brain, removal of ADO occurs largely via the astrocyte-based enzyme ADO kinase (ADK) (11-13). If metabolic clearance of ADO through ADK is impaired, SAH levels rise (10). SAH in turn is known to inhibit DNMTs through product inhibition (14).

$\mathrm{ADO}$ is an endogenous anticonvulsant in the brain (15) acting via activation of pre- and postsynaptic $A D O A_{1}$ receptors $\left(A_{1} R\right)$ to decrease neuronal excitability $(16,17)$. The ambient tone of $\mathrm{ADO}$ is determined by neuronal $\mathrm{ADO}$ release (18) and ADK-driven reuptake through equilibrative nucleoside transporters in astrocytes, which form a "sink" for ADO (19). Since disruption of ADO homeostasis and ADO deficiency has been implicated in epileptogenesis, local therapeutic $\mathrm{ADO}$ augmentation is an effective strategy to acutely 
A Transmethylation reaction S-adenosylmethionine (SAM)

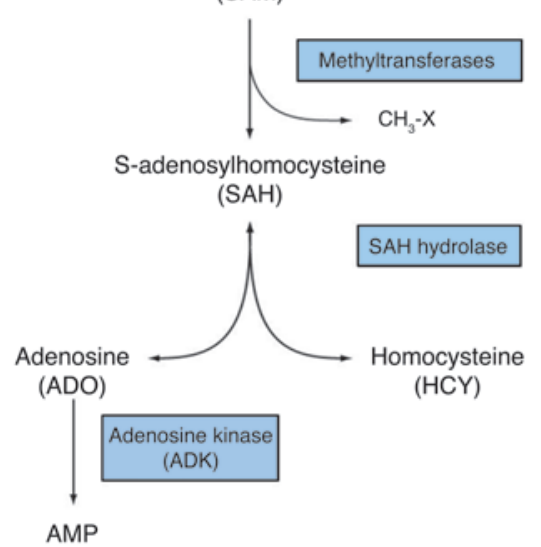

AMP
B

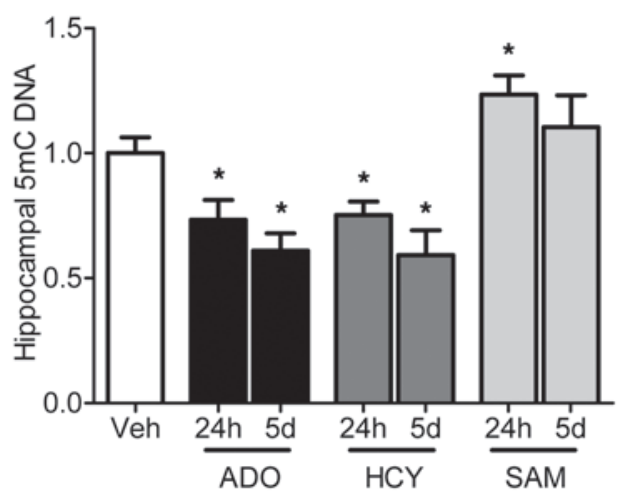

C

Genetic disruption
of ADK in mice

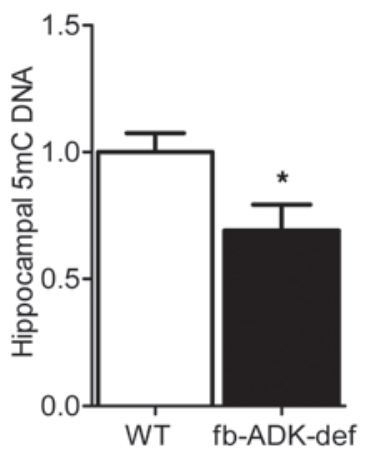

D $\quad A D K$ inhibitor

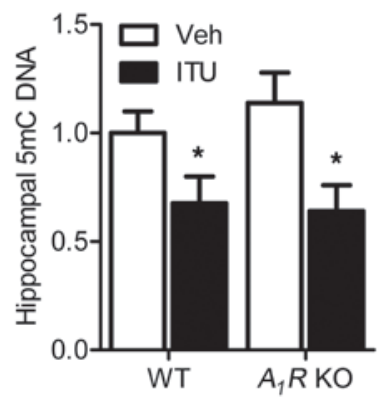

E $\quad$ ADK inhibitor + caffeine

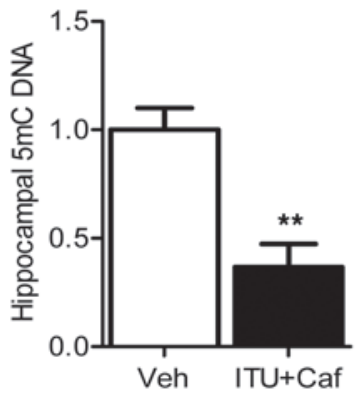

$\mathbf{F}$

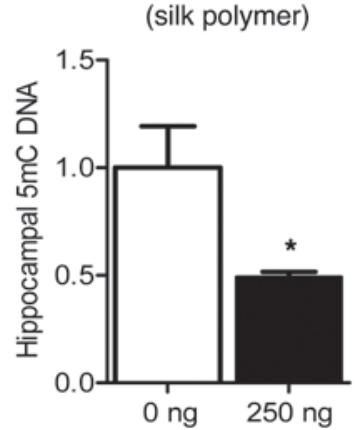

G Overexpression of ADK

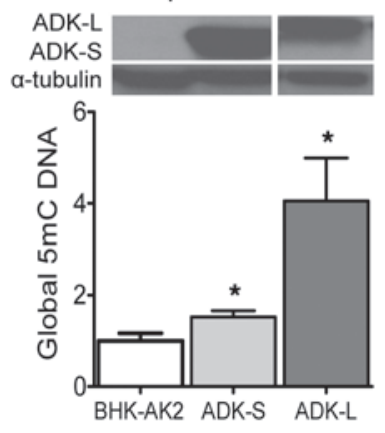

Figure 1

ADO regulates DNA methylation through interference with the transmethylation pathway. (A) Biochemistry of the transmethylation reaction. (B) A single i.c.v. bolus of ADO $(5 \mu \mathrm{g})$ or HCY $(250 \mu \mathrm{g})$ decreases global DNA methylation in the hippocampus at 24 hours and 5 days following the injection, whereas SAM $(16 \mu \mathrm{g})$ increases global DNA methylation at 24 hours. (C) Reduced ADK expression leads to a decrease in hippocampal DNA methylation in vivo as seen in transgenic fb-Adk-def mice with a forebrain-selective reduction of ADK. (D) The ADK inhibitor 5-ITU (3.1 mg/kg, i.p. once daily for 5 days) reduces global DNA methylation in hippocampus of WT and homozygous ADO A1R-KO mice. (E) Coadministration of the nonselective ADO receptor antagonist caffeine (Caf, $25 \mathrm{mg} / \mathrm{kg}$, i.p.) and ITU reduces hippocampal DNA methylation in WT mice. (F) Intraventricular ADO-releasing silk ( $250 \mathrm{ng} / \mathrm{d}$ ) decreases hippocampal DNA methylation in naive rats 5 days after implantation. (G) Overexpression of ADK leads to DNA hypermethylation in ADK-deficient BHK cells (BHK-AK2). Western blot shows protein expression from 3 pooled experimental replicates of BHK-AK2 cells transfected with the cytoplasmic (ADK-S) or nuclear (ADK-L) isoform of ADK and nontransfected control cells. Quantification of DNA methylation was assessed using 3 experimental replicates. ADK-L increases DNA methylation to a greater extent than ADK-S. Data are displayed as average \pm SEM. ${ }^{*} P<0.05 ;{ }^{* *} P<0.01 . n=4-9$.

suppress seizures in modeled epilepsy (20). However, possible epigenetic effects of ADO augmentation in the treatment of epilepsy, including the potential to modulate DNA methylation status, have not been studied to date. Based on ADO's role as an obligatory end product of DNA methylation, we hypothesized that an increase in $\mathrm{ADK}$ and the resulting decrease in $\mathrm{ADO}$, as seen in chronic epilepsy $(21,22)$, would lead to an increase in global DNA methylation in the brain. Further, we hypothesized that therapeutic ADO augmentation might be an effective strategy to reverse this pathological DNA hypermethylation and thereby prevent the progression of epilepsy.

\section{Results}

Increased $A D O$ and reduced $A D K$ expression induce DNA bypomethylation in the brain via interference with the transmethylation pathway. To provide mechanistic evidence that ADO contributes to the regulation of DNA methylation in the brain, we used a variety of techniques to manipulate $\mathrm{ADO}$. To identify the role metabolic interme- diates play in vivo to regulate DNA methylation, we administered a single intracerebroventricular (i.c.v.) bolus of either ADO, $\mathrm{HCY}$, or SAM (Figure 1, A and B). ADO and HCY, both end products in the transmethylation pathway, significantly decreased global DNA methylation in the hippocampus within 24 hours, an effect that was maintained for at least 5 days after infusion (Figure 1B). Conversely, injection of SAM, the primary methyl donor for transmethylation reactions, transiently increased global methylation by $24 \%$ at 24 hours (Figure $1 B$ ).

We next investigated whether changes in endogenous ADK expression might modulate DNA methylation in the brain. First, we examined transgenic mice (fb-Adk-def mice) with a forebrainselective reduction of ADK expression (21). We predicted that the resulting 3.3-fold increase in hippocampal ADO concentration (23) would suppress transmethylation and result in decreased DNA methylation. Indeed, a significant $31 \%$ decrease in global DNA methylation was seen in hippocampal isolates from fb-Adk-def 

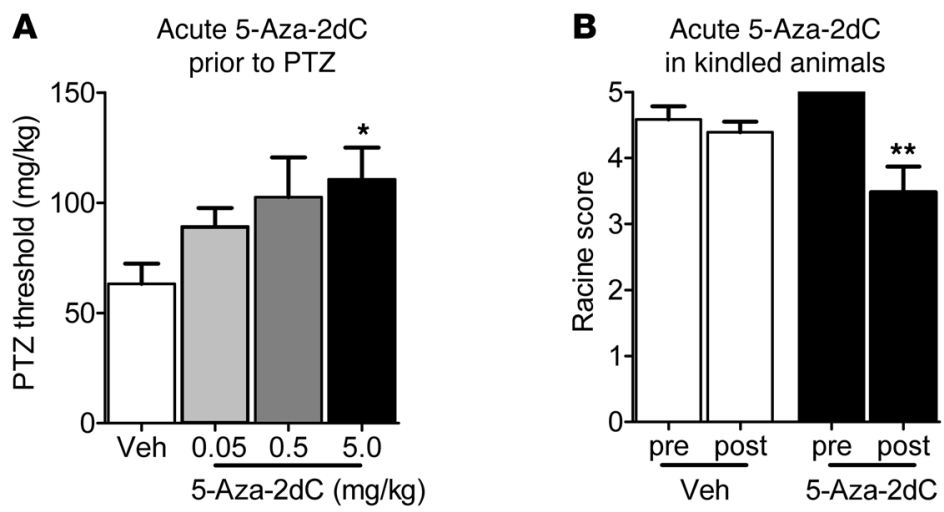

C Chronic 5-Aza-2dC during kindling
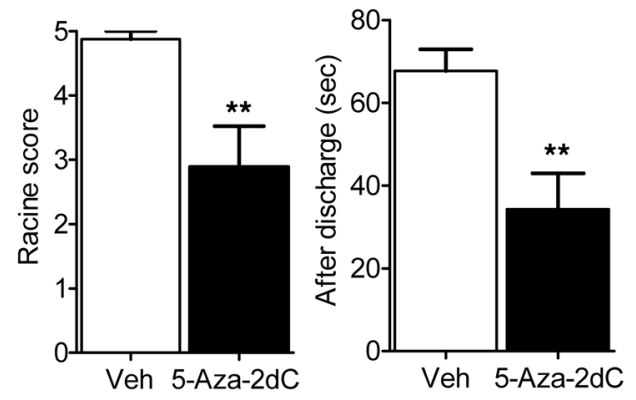

Figure 2

The DNMT inhibitor 5-Aza-2dC attenuates ictogenesis and epileptogenesis. (A) Dose response of WT mice pretreated with 5-Aza-2dC (0.05, $0.5,5.0 \mathrm{mg} / \mathrm{kg}$ ) 10 minutes prior to continuous PTZ infusion shows a significant increase in seizure threshold with the high-dose 5-Aza-2dC $(5.0 \mathrm{mg} / \mathrm{kg}$ ) compared with saline-treated controls. (B) A single injection of 5-Aza-2dC administered to fully kindled rats significantly reduces the average Racine score in subsequent stimulations (post-5-Aza-2dC) compared with the average Racine score prior to 5-Aza-2dC treatment (pre-5-Aza-2dC). There was no difference in the average Racine score in the rats prior to or following vehicle injection. (C) Rats kindled while being chronically treated with 5-Aza-2dC have a significant decrease in the average Racine score and after discharge duration compared with control kindled rats in response to a test stimulation administered after an 11-day stimulus- and drug-free period. Data are displayed as the average \pm SEM. ${ }^{*} P<0.05 ;{ }^{* *} P<0.01 . n=6-9$.

mice (Figure 1C). Likewise, chronic administration of the ADK inhibitor 5-iodotubercidin (5-ITU) $(3.1 \mathrm{mg} / \mathrm{kg}$ i.p. once every day, for 5 days) led to a significant (35\%) decrease in global DNA methylation in the hippocampus of WT mice (Figure 1D). Importantly 5-ITU-dependent hypomethylation was maintained in mice with a genetic disruption of the $\mathrm{ADO} \mathrm{A}_{1}$ receptor $(A 1 R \mathrm{KO})$, indicating that activation of the key receptor responsible for the anticonvulsant effects of ADO (24) is not required for the induction of ADOinduced hypomethylation (Figure 1D). To further demonstrate the biochemical basis of methylation interference (Figure 1D) and independence of ADO receptors, we coadministered the nonselective ADO receptor antagonist caffeine with ITU, which likewise resulted in a robust decrease in hippocampal DNA methylation (Figure 1E). Together, these findings show that modulating ADO tone either directly or via modulation of ADK expression can affect DNA methylation status in the hippocampus. In addition, our findings demonstrate what we believe is a novel ADO receptor-independent function of ADO, which acts by direct biochemical interference with the transmethylation pathway.

The nuclear isoform of ADK plays a key role in the induction of DNA bypermethylation. Mammalian ADK exists in 2 alternatively spliced isoforms, ADK long (ADK-L) and ADK short (ADK-S), which reside in the nucleus and cytoplasm, respectively (25). To investigate whether the nuclear isoform of ADK plays a unique role in the regulation of DNA methylation, we transfected cultured Adkdeficient BHK-AK2 cells (26) separately with an expression plasmid for either ADK-L or ADK-S and quantified global DNA methylation. Compared with the parental BHK-AK2 cells, recipients of ADK-L showed a robust $400 \%$ increase in global DNA methylation, whereas recipients of ADK-S showed only a modest $50 \%$ increase in global DNA methylation (Figure 1G). These results demonstrate that, while increases of both isoforms of ADK lead to increases in global DNA methylation, the nuclear isoform appears to be more effective in the regulation of DNA methylation status, suggesting the existence of cell-autonomous (nuclear ADK) and non-cellautonomous (cytoplasmic ADK) effects of ADK.
Therapentic delivery of ADO modulates DNA methylation. To investigate the therapeutic potential of ADO, we used ADO-releasing silk-based polymer implants to alter DNA methylation. We previously generated and characterized silk-based biodegradable brain implants able to deliver local doses of 8 to $1000 \mathrm{ng}$ ADO per day $(27,28)$. These implants successfully suppressed seizures in kindled rats (27) with no adverse effects. Here, we used implants designed to release a controlled dose of $250 \mathrm{ng}$ ADO per implant per day during a restricted time frame of 10 days (27). Five days following bilateral intraventricular implantation of ADO-releasing polymers in naive animals, global DNA methylation was significantly reduced (by $51 \%$ ) in the hippocampus when compared with that of animals receiving control polymers (Figure $1 \mathrm{~F}$ ). These data suggest that ADO-releasing polymers could be used as a therapeutic delivery device to modulate DNA methylation in vivo.

Inbibition of DNA methylation attenuates seizures and kindling-induced epileptogenesis. We have previously shown that increased ADK expression and the resulting decrease in ADO tone within the cortex and hippocampus are sufficient triggers for spontaneous focal seizures independent of an acute injury (21). Here, we establish that these conditions contribute to increased DNA methylation (Figure 1). Thus, we sought to determine whether changes in DNA methylation contribute to seizure susceptibility and epilepsy development. To address this question, we first performed a dose response study with the DNMT inhibitor 5-Aza-2' deoxycytidine (5-Aza-2dC) in a timed pentylenetetrazol (PTZ) seizure threshold test. WT mice treated with the highest dose of 5-Aza- $2 \mathrm{dC}(5.0 \mathrm{mg} / \mathrm{kg}$, i.v. $)$ 10 minutes prior to continuous PTZ infusion had a significant delay in latency to the extensor phase of seizures (Figure 2A). Similarly, in fully kindled rats, an acute bolus of 5-Aza-2dC significantly attenuated the average Racine score to 3.5 compared with the reproducible Racine stage- 5 seizures induced either prior to drug injection (pre-5-Aza-2dC) or in vehicle-treated controls (Figure 2B). Next, we assessed whether inhibiting DNMT activity during kindling acquisition would suppress epileptogenesis. Rats that were treated with 5-Aza-2dC throughout the kindling paradigm 
A

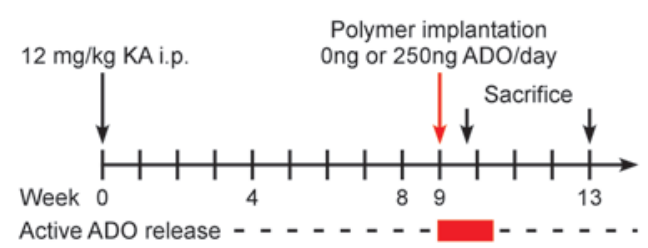

C

ADK densitometry

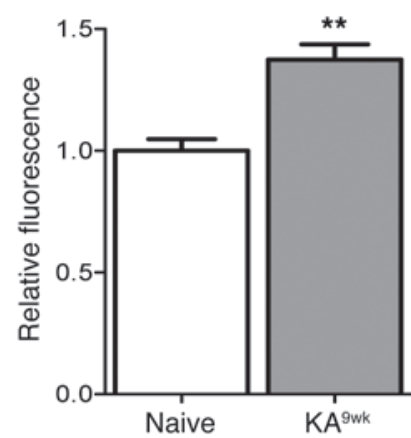

B

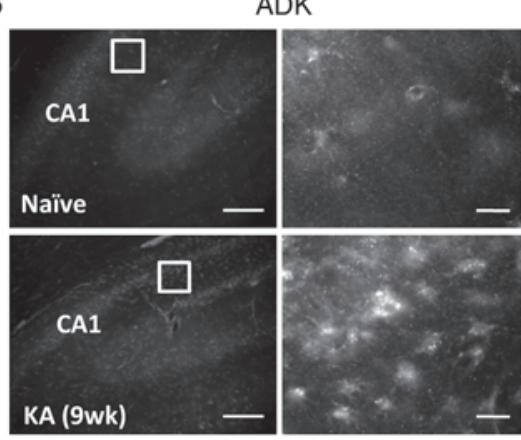

$5 \mathrm{mC}$ post $\mathrm{KA} \pm \mathrm{ADO}$

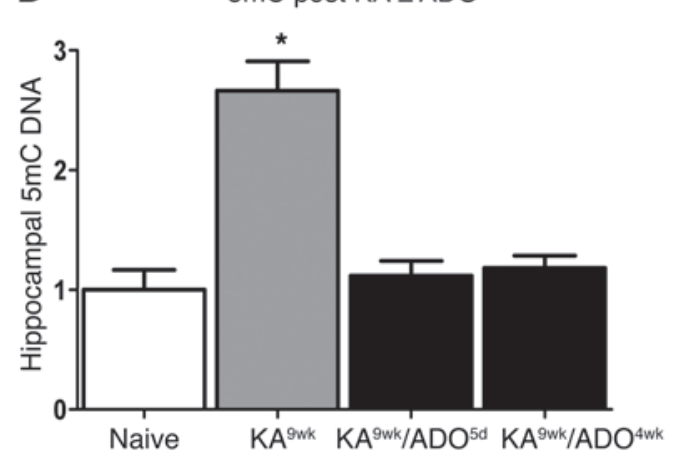

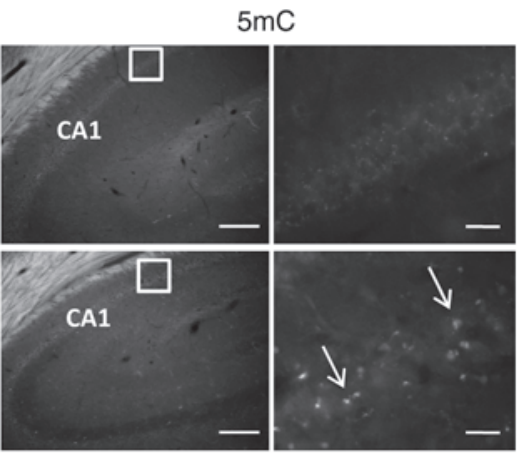

E DNMT post $K A \pm A D O$

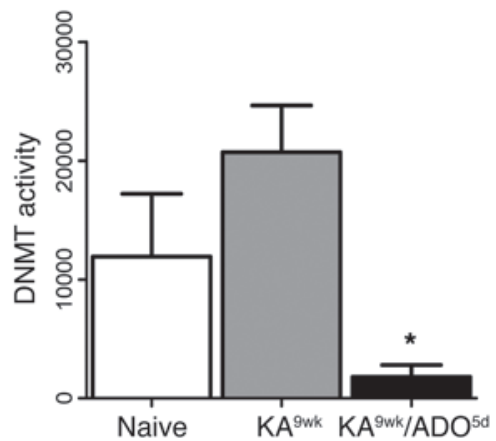

Figure 3

Intraventricular implants of ADO-releasing silk reverse DNA hypermethylation in the epileptic brain. (A) Experimental time line. (B) Hippocampal immunofluorescence images showing staining of ADK (left), and $5 \mathrm{mC}$ immunoreactivity (right) at low and high magnification in a naive rat (upper panels) and an epileptic rat at KA ${ }^{9 \mathrm{wk}}$ injection (lower panels). Note the regional match (in CA1) of ADK and $5 \mathrm{mC}$ (see arrows) immunoreactivity intensities. The white boxes in the low magnification images demarcate the region depicted at high magnification. Scale bars $=150 \mu \mathrm{m}$ and $15 \mu \mathrm{m}$ (low and high magnification, respectively). (C) Quantification of ADK immunofluorescence indicates a significant upregulation of ADK in the CA1 subregion of the hippocampus at KA ${ }^{\text {wwk }}$, compared with naive controls. (D) ADO-releasing silk attenuates the KA-induced increase in hippocampal DNA methylation in epileptic rats. Note that hippocampal DNA methylation, as quantified by ELISA, in ADO polymer-treated KA rats was reduced to naive levels. This reduction in hypermethylation persisted for at least 4 weeks following cessation of ADO release from the polymers. (E) DNMT activity increases in epileptic animals 9 weeks after systemic KA, an effect that was strongly and significantly suppressed by ADO released from the polymer. Data are displayed as average \pm SEM. ${ }^{*} P<0.05 ;{ }^{*} P<0.01 . n=3-8$.

had a significantly reduced average Racine score after receiving a single test stimulation compared with that of saline-treated controls. Furthermore, the average after-discharge duration was reduced by $51 \%$ in animals kindled in the presence of 5 -Aza- $2 \mathrm{dC}$ (Figure 2C). Although these experiments are limited to the use of only 1 DNMT inhibitor, which may also exhibit additional offtarget effects, these data suggest that inhibition of DNMT activity reduces seizure susceptibility and epilepsy acquisition.

Pathological ADK overexpression in the epileptic hippocampus correlates with DNA hypermethylation. Astrogliosis-associated increases in $\mathrm{ADK}$ expression and resulting ADO deficiency have been independently identified as pathological hallmarks of the epileptic brain $(21,22)$. Based on our findings linking the ADO tone to the global DNA methylation status, we predicted that increased ADK expression in epilepsy would lead to increased DNA methylation. To investigate this hypothesis, we employed a model of temporal lobe epilepsy (TLE) in rats characterized by the development of SRS triggered by systemic kainic acid-induced (KA-induced) status epilepticus (SE) (Figure 3A). Using immunohistochemical methods, we compared ADK and 5-methylcytidine $(5 \mathrm{mC})$ expression patterns found in the hippocampus of naive rats and rats sacrificed 9 weeks after the induction of epilepsy (Figure 3B). As predicted $(21,29)$, astrocytic
ADK immunoreactivity was increased throughout the hippocampal formation with highest increases (37\%) found near CA1 (Figure 3, $\mathrm{B}$ and $\mathrm{C}$ ). In line with increased $\mathrm{ADK}$ and reduced $\mathrm{ADO}$, we also found increased $5 \mathrm{mC}$ immunoreactivity in the epileptic hippocampus, most prominently seen in and near CA1 (Figure 3B). The spatial match of ADK overexpression with increased $5 \mathrm{mC}$ immunoreactivity suggests a functional interaction between ADK and DNA methylation status. Overexpression of ADK in astrocytes and DNA methylation changes in neurons suggests a non-cell-autonomous effect of ADO, which is also supported by our interference experiments with the transmethylation pathway (Figure 1).

Intraventricular implants of ADO-releasing silk reduce DNA bypermethylation in the epileptic brain. To determine whether transient ADO delivery could reduce DNA methylation in the epileptic brain, we implanted ADO-releasing polymers, which reduce DNA methylation in naive rats (Figure $1 \mathrm{~F}$ ), into the brain ventricles of epileptic animals at 9 weeks after KA (KA $\left.{ }^{9 \mathrm{wk}}\right)$ (Figure 3A). Global DNA methylation in whole hippocampal isolates was increased at $\mathrm{KA}^{9 \mathrm{wk}}$ injection compared with that in naive animals $(166 \% ; P=0.012)$ (Figure 3D). In contrast, on day 5 of ADO treatment, DNA methylation levels were restored to the naive state in epileptic rats with ADO polymer $(P=0.60)$ (Figure 3D). Importantly, this change 
A

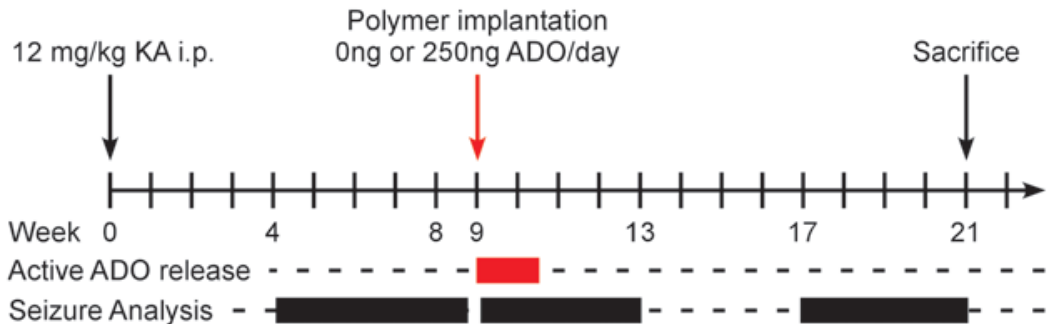

B Seizure frequency post $K A \pm A D O$ (silk polymer)

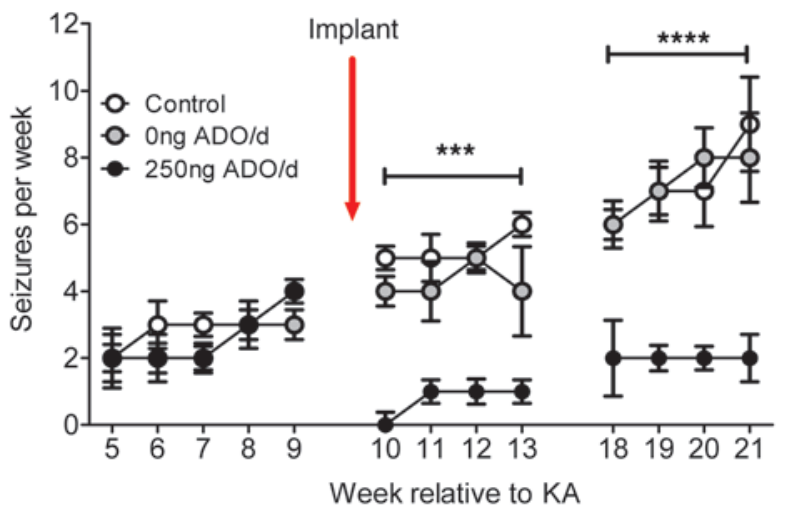

Representative EEGs post KA \pm ADO (silk polymer)

C

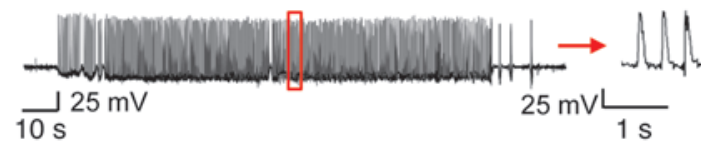

D

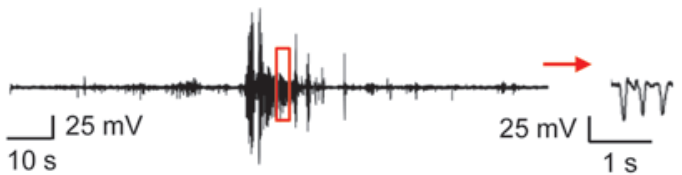

Figure 4

ADO-releasing silk polymers prevent progression of epilepsy development. (A) Experimental design. (B) Prevention of epileptogenesis by ADOreleasing silk. At KA ${ }^{9 \mathrm{wk}}$, epileptic rats received bilateral intraventricular implants of silk polymers releasing either $250 \mathrm{ng}$ (black circles) or $0 \mathrm{ng}$ (gray circles) ADO/d, or underwent sham surgery (white circles). Polymers transiently released a therapeutic dose of ADO for 10 days. Seizures were monitored by video analysis, and weekly seizure numbers are presented. Data are displayed as average \pm SEM, $n=8$ for ADO-implant recipients and 5-8 for control groups. Significant differences are represented between group comparisons (ADO vs. controls) as analyzed by a 2-way ANOVA. ${ }^{* \star} P<0.001$; ${ }^{* * \star} P<0.0001$. (C) Representative hippocampal EEG trace showing a 2-minute seizure in an animal that received control polymer (0 ng ADO/d) 9 weeks after systemic administration of KA. (D) Example of an epileptiform burst in an animal that received ADOreleasing polymer $(250 \mathrm{ng} / \mathrm{d})$ at $\mathrm{KA}^{9 \mathrm{wk}}$. Note, these events were rare in ADO-treated animals and lasted less than 15 seconds. Higher resolution traces (regions demarcated by red boxes in $\mathbf{C}$ and $\mathbf{D}$ ) are depicted to the right of the red arrows.

persisted for at least 3 weeks after cessation of ADO release from the polymers (4 weeks after implantation) (Figure 3D). These data suggest that a transient dose of ADO delivered locally can have a long-lasting effect on DNA methylation status. To understand the mechanism by which ADO augmentation changes DNA methylation status, we quantified the enzymatic activity of DNMT in epileptic rats. Nine weeks following the systemic injection of KA, DNMT activity in the epileptic animals was elevated almost 2-fold (174\%) compared with sham-injected nonepileptic control animals (Figure 3E), consistent with hypermethylation of hippocampal DNA in those animals (Figure 3, B and D). At 5 days of active ADO release, DNMT activity was almost completely blocked $(15 \% ; P<0.03)$ in the epileptic rats (Figure $3 \mathrm{E}$ ), consistent with restoration of normal DNA methylation status in these animals (Figure 3D).

ADO-releasing silk prevents progression of epilepsy development. Since seizure susceptibility and epilepsy development are partially dependent on changes in DNA methylation (Figure 2), we hypothesized that blocking pathological increases of DNA methylation with ADO therapy could halt long-term epilepsy progression. Because epileptogenesis is a lifelong process that continues after onset of the first SRS and leads to a progression in seizure frequency and severity (30), previous studies aimed at identifying antiepileptogenic drugs were frequently confounded by early initiation of treatment (1). Therefore, to rigorously test the antiepileptogenic potential of transient ADO therapy, we initiated treatment in "early epilepsy" after the onset of SRS using the systemic KA model of TLE (Figure 4A). Epilepsy progression was continuously monitored (24/7) from weeks 5-9 following systemic KA administration. Continuous epileptogenesis was reflected by a progressive increase in the number of seizures after initial SE (control animals, Figure 4B and ref. 31). Epileptic animals ( 9 weeks after SE; >10 SRS) subsequently received polymer implants that release ADO for a limited duration of 10 days (Figure 4A and ref. 27). Following polymer implantation, epilepsy progression was monitored in two 4-week recording sessions from weeks 10-13 and weeks 18-21 (Figure 4A). As expected, ADO-releasing polymers almost completely prevented any seizures during the first week following implantation (Figure 4B). Remarkably, reduced seizure activity was maintained far beyond the time window of active ADO release (first 10 days) up to at least 12 weeks after polymer implantation (75\% reduction of SRS incidence, $250 \mathrm{ng}$ vs. $0 \mathrm{ng} \mathrm{ADO} / \mathrm{d}$; $P<0.001$ ) (Figure 4B). Importantly, during weeks 18-21 following $\mathrm{KA}$, animals that were transiently exposed to ADO did not show a significant $(P>0.05)$ increase in seizure frequency, while control animals continued to worsen and 3 died due to excessive seizures. Together, these data demonstrate a potent antiepileptogenic role of transient focal ADO delivery. EEG recordings were performed in a separate cohort of animals to avoid potential confounds on DNA methylation analysis and histopathology. Those animals received intrahippocampal and cortical EEG recording electrodes during 
A

Mossy fiber sprouting post $\mathrm{KA} \pm \mathrm{ADO}$ (silk polymer)

21 weeks post $K A$
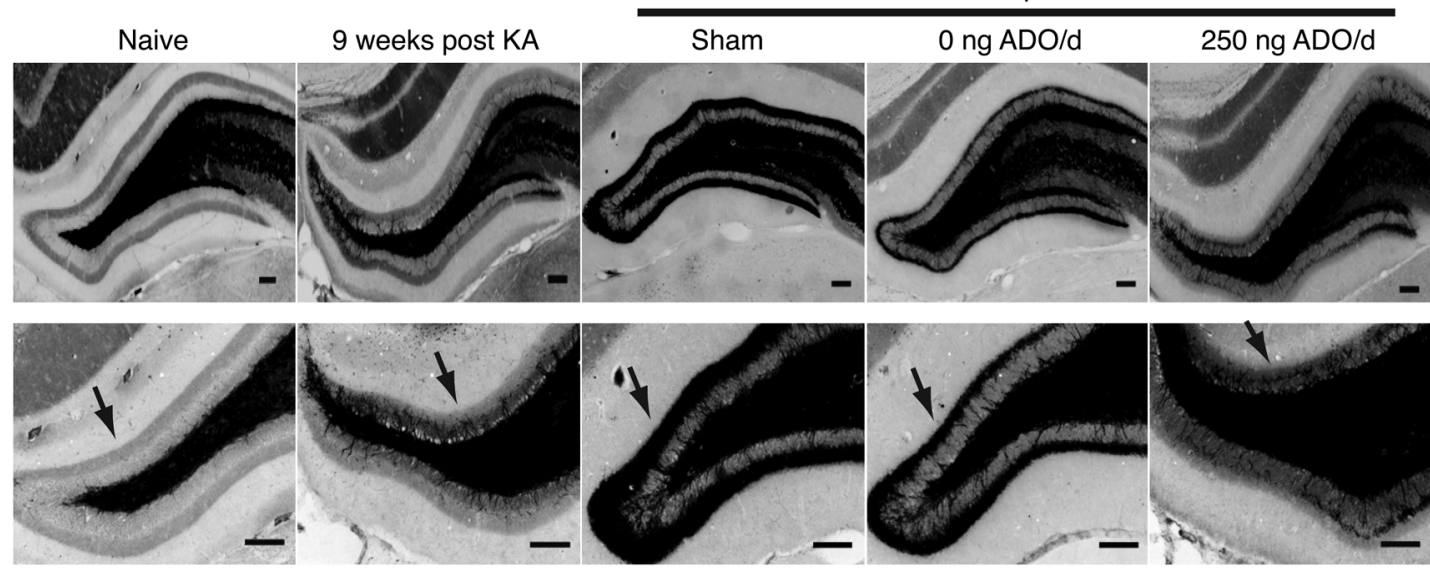

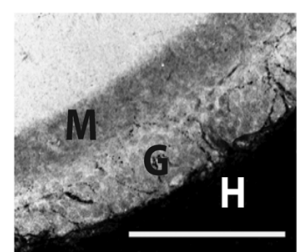

B
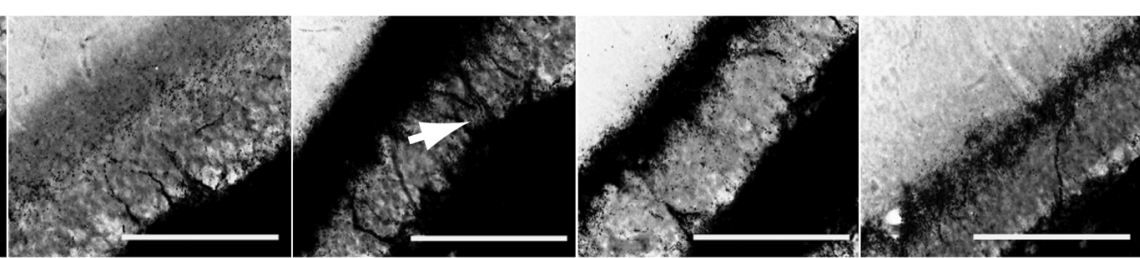

Mossy fiber quantification

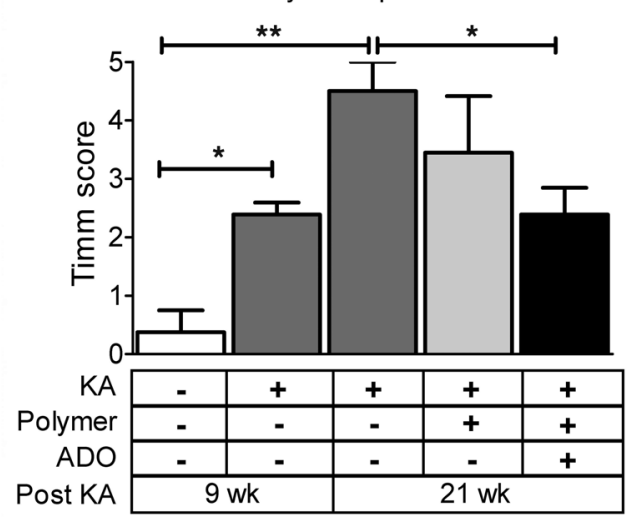

Figure 5

ADO-releasing silk implants prevent mossy fiber sprouting. (A) Transient ADO augmentation therapy in epileptic rats prevents mossy fiber sprouting in the dentate gyrus. Top row: representative low magnification images of Timm staining of mossy fibers in hippocampus in naive animals, $\mathrm{KA}^{9 \mathrm{wk}}$ animals, animals 21 weeks after KA (sham), and animals 21 weeks after KA that received polymers releasing either $0 \mathrm{ng}$ (control) or $250 \mathrm{ng} \mathrm{ADO} / \mathrm{d}$ for a duration of 10 days (implanted at KA ${ }^{9 \mathrm{wk}}$ ). Middle row: higher magnification images that depict Timm granules (black arrows) that correspond to mossy fiber synaptic terminals present in animals 21 weeks after KA (sham and $0 \mathrm{ng} \mathrm{ADO/d}$ animals but not in the $250 \mathrm{ng}$ ADO/d animals). Bottom row: high magnification images that illustrate extensive sprouting of mossy fiber axons in animals 21 weeks after KA (white arrow). M, molecular layer; G, granular layer; $H$, hilus. Original magnification, $\times 5$ (top row); $\times 10$ (middle row); $\times 40$ (bottom row). Scale bars: $500 \mu$ m. (B) Quantitative analysis of Timm staining shows that transient ADO delivery significantly reduced mossy fiber sprouting compared with that in animals 21 seconds after KA (sham) within the 3-month time span between weeks 9 and 21. Data represent group average Timm score in hippocampus and are displayed as mean \pm SEM. ${ }^{*} P<0.05 ;{ }^{*} P<0.01$. $n=3$ for all groups.

the polymer implantation surgery. Electrographic seizures were monitored in these animals from week 10-13 after KA. Whereas sham or control-polymer-receiving animals displayed robust seizures in the EEG (Figure 4C), seizure activity was markedly attenuated in recipients of the ADO-releasing silk polymers (Figure 4D). ADO-releasing silk implants prevent mossy fiber sprouting. To provide an independent outcome measure for the antiepileptogenic role of silk-based ADO delivery, we assessed the degree of granule cell axon (mossy fiber) sprouting (Figure 5A). Mossy fiber sprouting is thought to be a fundamental epileptogenic mechanism responsible for the formation of new recurrent excitatory circuits in the dentate gyrus (32). Nine weeks after SE, epileptic rats showed a significant increase in mossy fiber sprouting when compared with naive control animals, with visible axons beginning to spread from the hilar layer into the granular cell layer (Figure 5A). In sham-treated animals, mossy fiber sprouting was progressive; at 21 weeks after KA, axon sprouting increased and Timm granules, which correspond to mossy fiber synaptic terminals, presented 


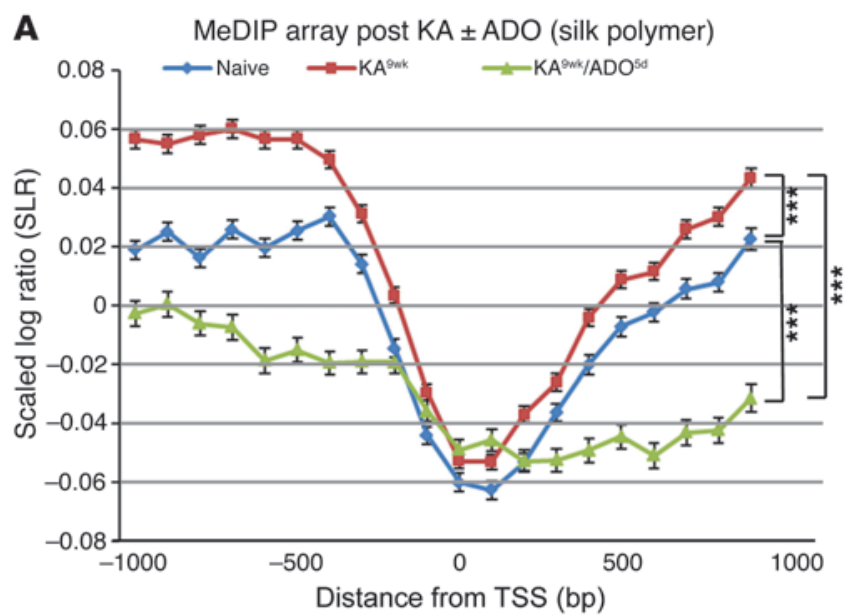

B Venn diagram for genes post $K A \pm A D O$ (silk polymer)

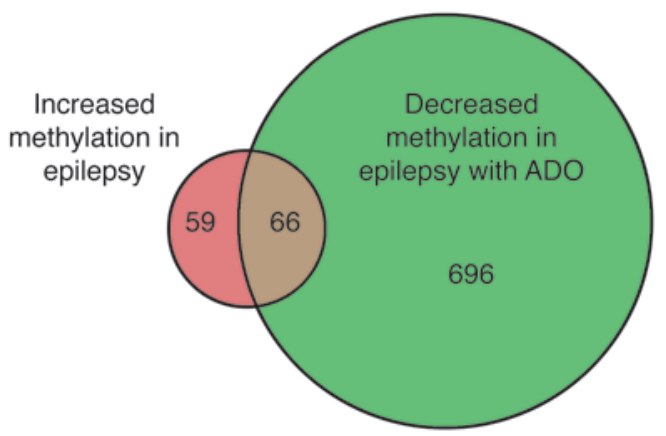

throughout the molecular layer of the dentate gyrus (Figure 5B). In stark contrast, animals that had transiently been exposed to ADO at 9 weeks after SE had no significant change in mossy fiber sprouting 12 weeks later (Figure 5, A and B). Thus, transient exposure to ADO prevented further mossy fiber sprouting, a major contributor to disease progression in epilepsy.

$A D O$ is a homeostatic regulator of global DNA methylation. Our data demonstrated that ADO homeostasis affects global DNA methylation through interference with the transmethylation pathway (Figure 1) in a non-cell-autonomous (Figure 1, B and F, and Figure 3B) and $\mathrm{ADO}$ receptor independent (Figure 1, D and $\mathrm{E}$ ) manner. Importantly, transient $\mathrm{ADO}$ augmentation also reduced global DNA methylation status in epileptic animals (Figure 3D). Our initial ELISAbased data sets demonstrate that ADO exerts homeostatic control over the global DNA methylation status. To validate those findings with an independent experimental strategy, we performed a methylated DNA immunoprecipitation on ChIP assay (MeDIP-onChIP) and compared the relative fraction of methylated and unmethylated genomic regions (log ratio) in pooled samples from $\mathrm{KA}^{9 \mathrm{wk}}$, rats exposed to 5 days of ADO delivery 9 weeks after KA $\left(\mathrm{KA}^{9 \mathrm{wk}} / \mathrm{ADO}^{5 \mathrm{~d}}\right)$, and naive rats. Array-wide, scaled log ratio (SLR) values were normally distributed and centered at 0 . We restricted our array analysis to probes within $1,000 \mathrm{bps}$ of a transcription start site (TSS) according to the Rat RGSC 3.4 assembly, as there was consistent probe representation on the array both up- and downstream of the TSS within this range. Within this range, we found that methylation nearest the TSS was lower in all experimental conditions (Figure 6A). On average, we found that the methylation status in the epileptic hippocampus, $\mathrm{KA}^{9 \mathrm{wk}}$ was higher than in naive hippo-

\section{Figure 6}

Whole-genome regulation of DNA methylation by ADO. (A) Genomewide analysis of hippocampal DNA extracts shows increased DNA methylation in the epileptic brain (red) and a robust decrease in DNA methylation in response to ADO treatment (green). Pooled ( $n=3-4$ / group) genomic DNA extracts from naive (blue), epileptic (KA ${ }^{9 \mathrm{wk}}$ ) and ADO-treated epileptic (KA $\left.{ }^{9 w k} / A^{5} O^{5 d}\right)$ rats were subjected to a MeDIP array followed by a comparison of the average \pm SEM SLR of all probes within $1000 \mathrm{bp}$ of the TSS. Note that with exception to the -100 to $100 \mathrm{bp}$ range, active ADO release reduces DNA methylation in the otherwise hypermethylated epileptic hippocampus. Repeated measures ANOVA (Statview) demonstrated a significant effect of the experimental group $(P<0.0001)$ and distance from TSS $(P<0.0001)$ as well as a significant interaction between experimental group and distance from TSS $(P<0.0001)$. Post hoc tests (Sheffe's $F)$ demonstrate that there are significant differences among the 3 experimental groups $\left({ }^{\star} P<0.0001\right)$. (B) Venn diagram for the number of genes where the DNA methylation is (a) increased in epilepsy (125; red) or decreased with ADO treatment (762, green); and (b) both increased in epilepsy and decreased in ADO treatment (66, overlap). The criterion for genes to be included in the Venn diagram is that $25 \%$ of probes within $\pm 1000 \mathrm{bp}$ of the TSS exceed a dSLR greater than 1.0 for $\mathrm{KA}^{9 \mathrm{wk}} \mathrm{vs.} \mathrm{naive} \mathrm{or} \mathrm{less}$ than -1.0 for $\mathrm{KA}^{9 \mathrm{wk} / A D O^{5 d}}$ vs. KA ${ }^{9 \mathrm{wk}}$.

campus (Figure 6A), consistent with our ELISA-based predictions (Figure $3 \mathrm{D}$ ). In the $\mathrm{ADO}$-treated epileptic rats $\mathrm{KA}^{9 \mathrm{wk}} / \mathrm{ADO}^{5 \mathrm{~d}}$, we found a uniform reduction in methylation (Figure 6A), again consistent with predictions from our ELISA-based results with ADO treatment alone (Figure 1B) and in the epileptic brain (Figure 3D). These results support our hypothesis that the epileptic brain is hypermethylated and that ADO treatment reduces methylation. To identify probes with the largest increase in methylation status in epileptic rats, we calculated the $\delta$ SLR (dSLR) between $\mathrm{KA}^{9 \mathrm{wk}}$ and naive control rats and the dSLR between $\mathrm{KA}^{9 \mathrm{wk}} / \mathrm{ADO}^{5 \mathrm{~d}}$ and $\mathrm{KA}^{9 \mathrm{wk}}$. We considered the methylation status of a probe to be significantly increased if the dSLR was greater than or equal to \pm 1 , a threshold chosen because it identified the top $2.5 \%$ of changed probes in our restricted data set (within 1,000 bps of each TSS). In the Nimblegen array, each TSS was associated with 11 to 20 probes. If at least $25 \%$ of the probes associated with a TSS had a $\mathrm{KA}^{9 \mathrm{wk}}$ vs. naive dSLR of 1 or more, we considered the associated gene to be a candidate for significantly increased methylation in the epileptic brain. Using these criteria, we identified 125 genes with substantially increased methylation in the epileptic brain. We demonstrated the phenotypic relevance of these DNA methylation changes in epileptic vs. control rats by comparison of mRNA expression changes from a published mRNA array data set (GEO GSE14763) consisting of pilocarpine-induced epileptic rats compared with controls. From our MeDIP array, we chose the 10 targets with the most positive dSLR values of genes also represented on the rat gene expression array (i.e., the "top 10 list" of targets with the most hypermethylated probes); 70\% of these genes (Unc13c, Exoc8, Mtmr14, Phkg2, Umps, Spata9, and Gsta5) indeed have decreased mRNA expression in epileptic versus control rats and thus confirm the prediction that increased DNA methylation relates to decreased gene expression. This comparison further validates the MeDIP array results. By similar criteria, comparison of $\mathrm{KA}^{9 \mathrm{wk}} / \mathrm{ADO}^{5 \mathrm{~d}}$ with $\mathrm{KA}^{9 \mathrm{wk}}$ rats and a resulting dSLR of $\leq-1$ or less, we identified 762 genes that showed reduced methylation status during ADO treatment. Sixty-six genes were identified as common within the 2 groups (see Supplemental Table 1; supplemental material available online with this article; 


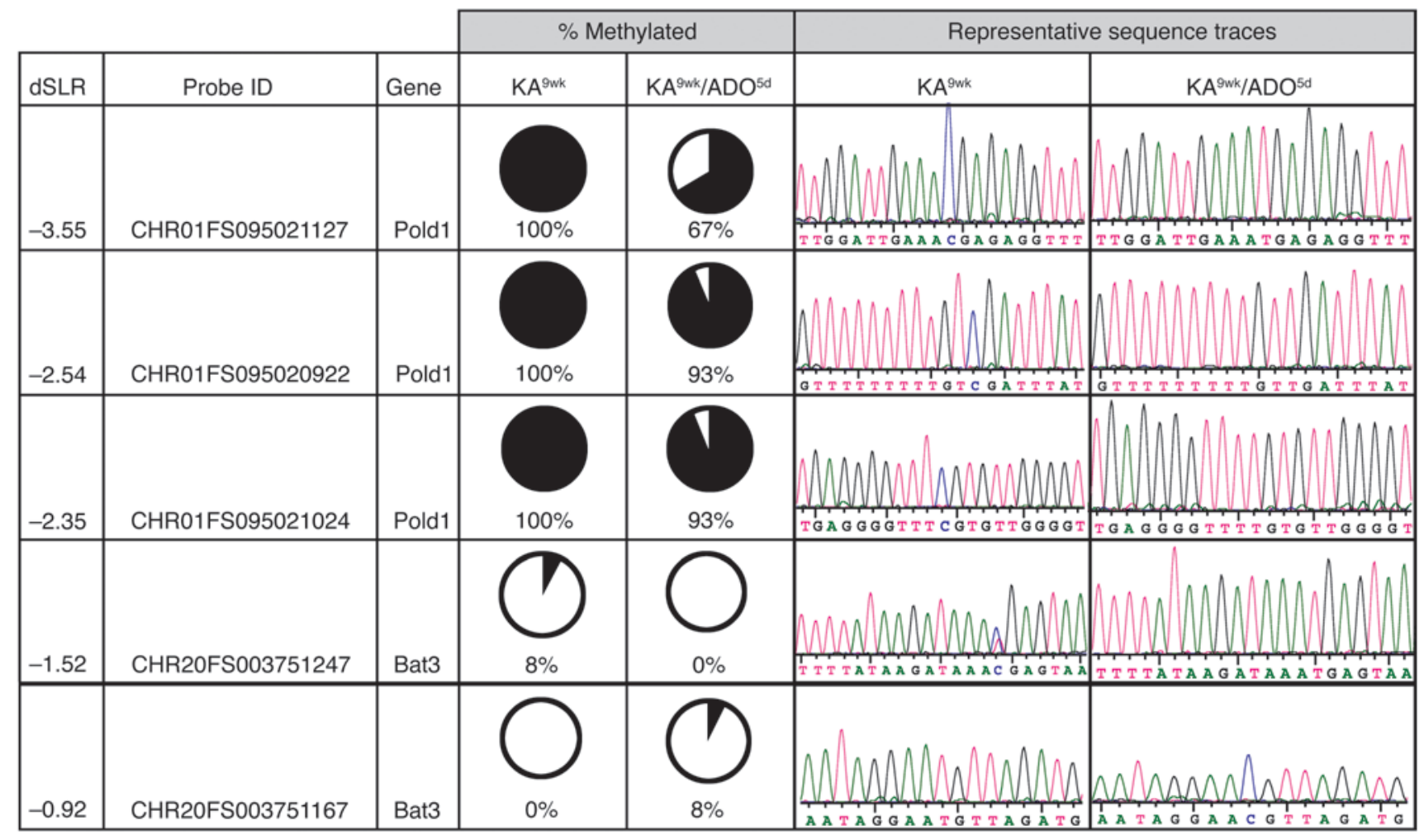

Figure 7

ADO treatment reduces genomic DNA CpG methylation in the epileptic hippocampus. Analysis of bisulfite sequencing of hippocampal DNA from epileptic and ADO-treated epileptic rats confirms that predictions from the MeDIP array are translated to changes in the methylation status of CpGs. Bisulfite sequencing was performed on DNA extracts from KA ${ }^{9 \mathrm{wk}}$ and KA ${ }^{9 \mathrm{wk}} / \mathrm{ADO}^{5 \mathrm{~d}}$ vs. KA ${ }^{9 \mathrm{wk}}$ rats $(n=3$ animals/treatment and 4-5 clones/animal). The methylation status of individual probes, which contain only $1 \mathrm{CpG}$ and which span a dSLR range from -0.92 to -3.55 $\mathrm{KA}^{9 \mathrm{wk}} / \mathrm{ADO}^{5 \mathrm{~d}}$ vs. KA ${ }^{9 \mathrm{wk}}$ was compared between treatment groups. Changes in methylation are displayed as percentage of methylated (pie chart, black corresponds to the percentage of methylated CpGs; $1 \mathrm{CpG}$ per probe; 12 to 15 clones total) and in the representative sequence traces with methylated cytosines retained (blue peaks) and unmethylated cytosines converted to thymine (pink peaks). Note that at a KA ${ }^{9 \mathrm{wk}} / \mathrm{ADO}^{5 \mathrm{~d}} \mathrm{vs}$. KA ${ }^{9 \mathrm{wk}}$ dSLR threshold of less than -3.0 , there is a robust decrease (33\%) in ADO-mediated CpG methylation in 3 out of 3 animals. Probes with a dSLR greater then a -3.0 threshold $(-0.92$ to -2.54$)$ yielded only slight variations $(8 \%)$ in the methylation status.

doi:10.1172/JCI65636DS1). In summary, these data demonstrate what we believe to be a novel function of ADO as a homeostatic regulator of global DNA methylation status, which - according to the underlying biochemistry (Figure $1 \mathrm{~A}$ ) - does not directly provide for target specificity. We demonstrate that global hippocampal DNA methylation increased during epileptogenesis and decreased following transient ADO treatment, validating our previous findings (Figure 3) in an independent experimental approach.

ADO treatment reduces genomic DNA CPG methylation in the epileptic bippocampus. The MeDIP array results predict that, within 1,000 bps of each TSS, DNA methylation throughout the genome is consistently increased in epileptic rats and decreased in ADO-treated epileptic rats (Figure 6A). To validate the general robustness of the MeDIP data set, bisulfite sequencing was conducted on genomic regions corresponding to a total of 5 individual probes that each contained only a single CPG site and that covered a wide representative range $(-3.5$ to -0.92$)$ on the $\mathrm{KA}^{9 \mathrm{wk}} / \mathrm{ADO}^{5 \mathrm{~d}}$ vs. KA ${ }^{9 \mathrm{wk}}$ dSLR (Figure 7 ). When comparing untreated and ADO-treated epileptic rats, probes with a more negative dSLR (i.e., -3.5) are expected to have a robust difference in the percent methylation. For each probe, the methylation status of its single CPG dinucleotide was compared between bisulfite converted hippocampal DNA from $\mathrm{KA}^{9 \mathrm{wk}}$ - and $\mathrm{KA}^{9 \mathrm{wk}} / \mathrm{ADO}^{5 \mathrm{~d}}$-treated rats ( $n=3$ animals/group and 4-5 clones/animal). Importantly, the greatest ADO-mediated reduction in percentage of methylation $(33 \%)$ was associated with the probe that had the largest negative dSLR value $(-3.55)$. This probe was associated with the gene PolD1; its CpG was $100 \%$ methylated in the $\mathrm{KA}^{9 \mathrm{wk}}$ rats, while we observed a $33 \%$ reduction in $\mathrm{CPG}$ methylation of this PolD1 probe in $\mathrm{KA}^{9 \mathrm{wk}} / \mathrm{ADO}^{5 \mathrm{~d}}$ rats. ADO dependent reduction of methylation was found in 3 out of 3 animals and in 1 to 3 out of 5 sequenced clones per animal. In contrast, 4 additional probes from 2 different genes (PolD1 and Bat3) that spanned an $\mathrm{KA}^{9 \mathrm{wk}} / \mathrm{ADO}^{5 \mathrm{~d}} \mathrm{vs}$. $\mathrm{KA}^{9 \mathrm{wk}} \mathrm{dSLR}$ range from -0.92 to -2.54 had CpG methylation changes of 7\%-8\% (i.e. in only 1 out of 12 to 15 sequenced clones) between $\mathrm{KA}^{9 \mathrm{wk}} / \mathrm{ADO}^{5 \mathrm{~d}}$ and $\mathrm{KA}^{9 \mathrm{wk}}$ rats. These data validate that ADO therapy causes decreased methylation in individual CPG sites at dSLR values of -1 or greater and demonstrate that dSLR values of -3 or greater predict robust decreases in DNA methylation across all animals and several clones. Thus, the magnitude of the $\mathrm{KA}^{9 \mathrm{wk}} / \mathrm{ADO}^{5 \mathrm{~d}} \mathrm{vs} . \mathrm{KA}^{9 \mathrm{wk}} \mathrm{dSLR}$ calculated from the MeDIP array positively correlates with a reduction in percentage of methylation in ADO-treated rats as confirmed by bisulfite sequencing. 


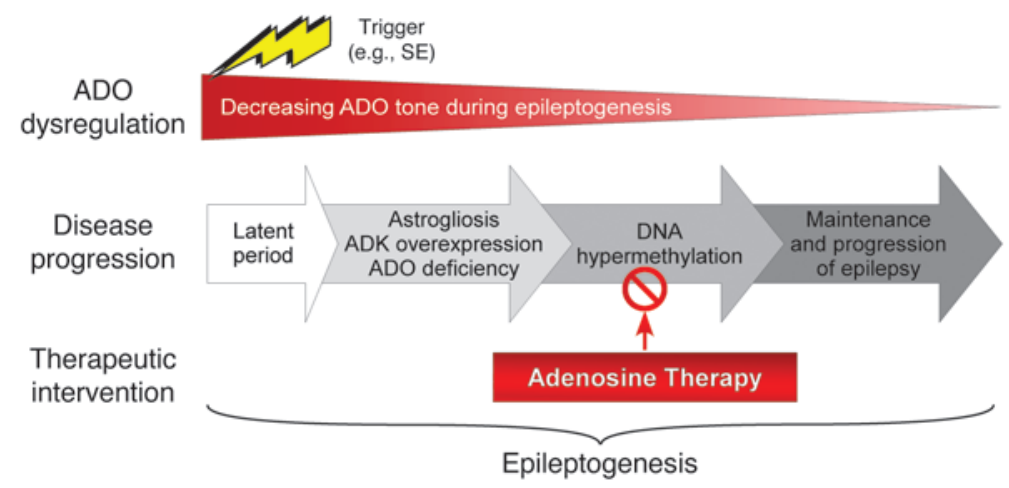

Figure 8

Model for the role of ADO and associated DNA methylation changes in epileptogenesis. As part of ongoing epileptogenesis astrogliosis with associated overexpression of ADK occurs, this results in subsequent ADO deficiency. These alterations lead to DNA hypermethylation and maintenance and progression of the epileptic phenotype. Therapeutic ADO augmentation interferes with DNA methylation and may interrupt this process. For details, see main text.

\section{Discussion}

In the present study, we identify what we believe is a novel epigenetic function of the purine ribonucleoside $\mathrm{ADO}$ as a homeostatic regulator of global DNA methylation. Our findings demonstrate that there is an increase in DNA methylation in the hippocampi of epileptic animals and that transient ADO therapy effectively reduces this pathological DNA methylation status. Remarkably, this transient ADO therapy also effectively prevents epileptogenesis. Previously, ADO augmentation has been well characterized as an $\mathrm{A}_{1} \mathrm{R}$-dependent anticonvulsant modality (19); however, these receptor-mediated effects are limited to the time period of therapeutic intervention (27). Here, we show that $\mathrm{ADO}$ tone can directly modulate DNA methylation in vivo and thereby exert additional epigenetic effects via biochemical interference with the transmethylation pathway. These changes affect the DNA methylome on a homeostatic level, are maintained long after therapy is suspended, are non-cell autonomous, and are ADO receptor independent. By broadly targeting homeostatic functions of multiple intracellular pathways via genome-wide changes in the DNA methylation status, we demonstrate here that ADO-induced changes in the DNA methylome in a global homeostatic sense could be used to attenuate disease progression in epilepsy. Together, these data ascribe a function to the brain's endogenous anticonvulsant ADO as a biochemical regulator of the methylome and directly support the "methylation hypothesis of epileptogenesis" (2).

The studies presented here show that local ADO augmentation via implantable biodegradable polymers can inhibit DNA methylation in the CNS of both healthy and epileptic animals. There is increasing evidence to support the idea that even a brief exposure to an epigenetic modulator may lead to long-lasting changes (33), which can best be explained by homeostatic network effects on the "epigenomic landscape." For example, recent work in cancer biology has shown that exposure to transient low doses of DNA demethylating agents results in long-term anti-tumor effects, modulated by genomewide promoter methylation, which persist well beyond drug withdrawal (34). In agreement with these studies, our transient biochemical manipulation has long-last- ing effects, preventing progression of epileptogenesis for at least 3 months in a model of mesial TLE. The antiepileptogenic effect of transient ADO delivery was documented in 2 independent outcome measures. First, we demonstrated that transient ADO delivery resulted in a sustained reduction of seizures over a time span of at least 3 months, during which all control animals continued to progress in seizure intensity (Figure 4). Second, we demonstrated a suppression of mossy fiber sprouting, a well-recognized pathophysiological phenomenon of TLE (ref. 32 and Figure 5).

To examine the role ADO plays in affecting methylation homeostasis on the network level, we followed 2 independent experimental approaches. Using an ELISAbased assay as well as a rat-specific MeDIP-on-ChIP analysis, we compared the global methylation state of hippocampal DNA derived from experimentally naive rats with that of untreated epileptic rats as well as with that of epileptic rats treated with an ADO-releasing silk polymer for 5 days. In both assays, we found an increase in global DNA methylation status in epileptic rats versus nonepileptic control rats (Figure 3D and Figure 6A). Importantly, 5 days of exposure to ADO reduced the global DNA methylation status in epileptic animals (Figure 3D and Figure 6A). These data independently demonstrate that ADO provides homeostatic regulation of the "DNA methylation landscape." This novel function of ADO is consistent with the underlying biochemistry (Figure 1A), which does not provide any mechanism for target specificity. These homeostatic control functions are also consistent with a non-cell-autonomous effect of ADO (Figure $1 \mathrm{~B}$ and Figure $3 \mathrm{~B}$ ) and with independence from $\mathrm{ADO}$ receptor activation (Figure 1, D and E). Although not the direct focus of our study, the control of methylation homeostasis by ADO does not exclude the possibility for targeting specific changes afforded by ADO therapy. Among the targets that showed reduced DNA methylation during active ADO release (Supplemental Table 1), several specifically interact with DNA or play a role in gene transcription and translation (PolD1, Polr1e, Rps6kl1, Snrpn, Znf524, Znf541, Znf710), making them likely candidates as mediators for ADO-dependent changes in major homeostatic functions. Although individual targets need to be validated in additional studies, our conceptual MeDIP predictions have been validated with bisulfite sequencing, which confirms that ADO-mediated changes in global DNA methylation (ELISA and MeDIP) are directly attributable to site-specific changes in genomic DNA CPG methylation. Furthermore, we selected a CpG site from PolD1, a gene that encodes a component of the DNA polymerase $\delta$ complex, as an illustrative example for a $\mathrm{CpG}$ site that displays a robust ADO-mediated site-specific change in the DNA

Table 1

Target and primer information for bisulfite sequencing

$\begin{array}{ccccc}\text { Gene } & \begin{array}{c}\text { Product } \\ \text { size }(\mathbf{n t})\end{array} & \text { Tm } & \text { Direction } & \text { Primer sequence } \\ \text { PolD1 } & 384 & 57.7 & \begin{array}{c}\text { Left } \\ \text { Right }\end{array} & \begin{array}{c}\text { GGAGGTTAAAGATATATTTGGGATTT } \\ \text { TCTCTCTATAAACAAAACAACTAC }\end{array} \\ \text { Bat3 } & 233 & 58.1 & \begin{array}{c}\text { Left } \\ \text { Right }\end{array} & \begin{array}{c}\text { GGTGAGGATAGGTAAATAAATTTGTT } \\ \text { TAATAAAACCCTAAACTCTCAAACTC }\end{array}\end{array}$

Tm designates the melting temperature $\left({ }^{\circ} \mathrm{C}\right)$ of the primers. 
methylation status. The magnitude of the $\mathrm{KA}^{9 \mathrm{wk}} / \mathrm{ADO}^{5 \mathrm{~d}} \mathrm{vs} . \mathrm{KA}^{9 \mathrm{wk}}$ dSLR calculated with the MeDIP data set also positively correlated with reduced methylation, adding confidence in the MeDIP predictions (Figure 7). Although not validated by independent replicates on a target basis, the results of our MeDIP-on-ChIP array suggest that a large number of genes associated with DNA structural elements and transcription factors are altered by ADO treatment in the epileptic brain; thus, the protective effect of ADO augmentation might not be mediated via a single gene, but through a network of gene expression changes.

ADO treatment is not expected to decrease methylation at all CpG sites at the same time. Whereas site-specific methylation is regulated by targeting DNMT complexes by a variety of mechanisms (35), a change in the ADO concentration shifts the equilibrium constant of the transmethylation pathway, thereby either permitting or preventing the act of DNA methylation. Within the epileptic brain, high ADK and low ADO will drive SAM methyl group donation to DNA, resulting in a pathological hypermethylation status. Conversely, increasing ADO levels with the silk polymer shifts the equilibrium constant to preventing methylation and restores normal methylation levels in the epileptic brain. At this point, we therefore do not propose the direct involvement of active DNA demethylation. However, since ADK overexpression drives DNA hypermethylation (Figure 1G), we cannot rule out the possibility that the epileptic brain compensates to a certain degree by upregulation of active DNA demethylation. Importantly, dynamic demethylation has been demonstrated in neurons in response to experimentally induced seizures, in which hippocampal $B d n f$ and $F g f 2$ were both rapidly demethylated in a GADD45B-specific manner (5). GADD45B expression was shown to rise as an acute response to electrical stimulation, suggesting that seizures trigger a transient increase of GADD45B and thus promote active demethylation (36). Our data show that during active ADO delivery, DNA methylation status is rapidly reduced within 5 days of ADO treatment (Figures 3, 6, and 7). This reduction of the DNA methylation status following ADO treatment is compatible with blockade of DNA methylation through product inhibition of DNMTs (ref. 14; see also suppression of DNMT activity after 4 days of ADO exposure; Figure 3E), likely under conditions of increased compensatory DNA demethylation, an intriguing possibility that warrants further investigation.

We previously demonstrated that dysregulation of ADO homeostasis due to overexpression of the key ADO-metabolizing enzyme ADK leads to exacerbation of epilepsy $(21,37)$. Similarly, kindling epilepsy was associated with a loss of adenosinergic control mechanisms, in particular with a decrease of $A D O A_{1} R$ densities in the epileptic brain (38). In light of the epigenetic findings presented herein, we propose a refined model of the ADK hypothesis of epileptogenesis (ref. 39 and Figure 8). Once the epileptic phenotype is established with overt astrogliosis, overexpression of ADK, and $\operatorname{ADO}$ deficiency $(21,22,37)$, there is a pathological hypermethylation of DNA. Increased methylation in the epileptic brain in turn is thought to perpetuate and to exacerbate epileptogenesis (2). As we have demonstrated here, transient ADO therapy might prevent perpetuation of ongoing epileptogenic processes (Figure 8) by intervening with biochemical mechanisms that maintain the hypermethylated state in epilepsy. We cannot exclude, however, the possibility that ADO may have additional distinct effects on $\mathrm{ADO}$ receptor expression, an intriguing possibility that warrants further investigation.
It is important to note that intracellular changes in ADK expression within astrocytes may have both cell-autonomous and noncell-autonomous ramifications. ADO levels within astrocytes and neurons are regulated by equilibrative and concentrative nucleoside transporters $(40,41)$. Thus, an increase in ADK in astrocytes, as observed in the epileptic hippocampus, may directly affect DNA methylation within the affected astrocyte (cell-autonomous function). Additionally, a pathological increase of ADK in astrocytes reduces the global ADO tone through the transport and metabolism of extracellular ADO to AMP, thereby indirectly modulating the activity of neighboring cells (i.e., epigenetic changes in neurons). This non-cell-autonomous effect is supported by our findings that increased astroglial ADK expression in epileptic rats leads to increased $5 \mathrm{mC}$ immunofluorescence in adjacent neurons (Figure 3).

In order for a new antiepileptogenesis intervention to be clinically relevant, the window of effectiveness is particularly important. Previous reports on "antiepileptogenesis" were based on early intervention within hours, or at most a few days, before or after an epileptogenesis-precipitating injury. Even though partial antiepileptogenic effects were reported in some studies, it is not clear whether epileptogenesis was truly suppressed or whether the precipitating injury was modified (1). Our present study differs because we delayed therapeutic intervention until all animals developed "early epilepsy" (at least 10 SRS); thus, we were able to monitor long-term disease progression (i.e., epileptogenesis) without any confounds related to injury modification.

When considering how to advance ADO-based therapies to clinic applications, safety and feasibility must be taken into consideration. Following surgical resection of an epileptogenic focus, seizures recur in about $50 \%$ of patients and secondary epileptogenesis is a major problem (42). Placement of ADO-releasing silk into the resection cavity following epilepsy surgery might be used as preventative treatment. Similarly, transient ADO delivery might be used as prophylaxis in patients at risk for developing epilepsy, e.g., following a severe traumatic brain injury. Finally, since epileptogenesis is a lifelong ongoing process in patients with epilepsy, local treatment with ADO-releasing silk might be envisioned as a feasible therapeutic strategy for preventing disease progression with its sequelae of comorbidities and pharmacoresistance.

\section{Methods}

Animal models ofepilepsy. For seizure threshold testing, male CD-1 mice (25-35 g) were used. $0.75 \%(\mathrm{w} / \mathrm{v}) \mathrm{PTZ}$ was prepared in isotonic saline. 5-Aza-2dC was prepared in isotonic saline and administered via tail vein 10 minutes prior to initiation of PTZ infusion. PTZ was infused via tail vein at a rate of $0.2 \mathrm{ml} / \mathrm{min}$ using a Hamilton microsyringe (Harvard Apparatus) in freely moving animals. Times to first twitch, first clonus, and final extensor phase were recorded. Infusion was stopped after a final extension or at a maximum volume of $0.9 \mathrm{ml}$, whichever came first.

For the kindling model and associated 5-Aza-2dC drug testing, male Sprague Dawley rats (300-350 g) were implanted with bipolar, coated stainless steel electrodes $(0.20 \mathrm{~mm}$ in diameter; Plastics One) into the right hippocampus (stereotactic coordinates relative to Bregma: AP, $-4.2 \mathrm{~mm}$; ML, -4.6 ; DV, -5.6). For experiment 1, the animals were kindled based on a rapid kindling protocol (43). Briefly, using a Grass S-88 stimulator, rats received 12 stimulations per day (1-ms square wave pulses of $200 \mu \mathrm{A}, 50-\mathrm{Hz}$ frequency, 10 s duration at an interval of 30 minutes between stimulations) every 2-3 days until a stable Racine stage-5 seizure was generated. Following a 2-day stimulus-free period, rats received 2 stimulations, then a single i.p. bolus of either saline or $5-\mathrm{Aza}-2 \mathrm{dC}(0.5 \mathrm{mg} / \mathrm{kg}) 15$ minutes prior to a subsequent series of 
9 stimulations. All stimulations were delivered at an interval of 30 minutes, and rats were scored for mean Racine score and after-discharge duration. There was a 5-day drug- and stimulus-free period between the saline and 5-Aza-2dC trials. For experiment 2 , animals were kindled as described above while being chronically treated with either saline or $5-\mathrm{Aza}-2 \mathrm{dC}(0.4 \mathrm{mg} / \mathrm{kg}$, i.p.) administered 12 hours prior to the first kindling session and every successive 12 hours until the saline-injected controls achieved a stable Racine stage-5 seizure score. Following an 11 day drug- and stimulus-free period, animals received a stimulus and were scored for seizure stage and duration.

For the TLE model, young male Sprague Dawley rats (130-150 g) received a single acute dose of KA (12 mg/kg, i.p.) to trigger SE. Only rats that exhibited at least 3 hours of convulsive Racine stage- 4 seizures were used. Starting 4 weeks after KA administration, animals were continuously video monitored to quantify the number of convulsive stage $4-5$ seizures per week. The number of stage 4-5 seizures typically increased to more than 3 seizures per week at $\mathrm{KA}^{9 \mathrm{wk}}$, and animals experienced at least 10 spontaneous convulsive stage 4-5 seizures (inclusion criterion). All animals were further monitored during weeks $10-13$ and 18-21 after KA administration. Behavioral seizures were confirmed by EEG analysis in selected animals, with bipolar recording electrodes implanted into the right hippocampus (AP, $-4.2 \mathrm{~mm}$; ML, -3.0; DV, -3.3). Electrical brain activity was amplified (Grass Technologies) and digitized (PowerLab; AD Instruments). EEG files were analyzed manually by an observer blinded to the animals' treatment. EEG seizure activity was defined as high-amplitude rhythmic discharges that clearly represented a new pattern of tracing that lasted at least 5 seconds.

Polymer implant design and implantation. Cylindrical silk-based polymer implants designed to deliver the target dose of $250 \mathrm{ng}$ ADO per day for a limited period of 10 days were designed and fabricated as described $(27,28)$. Consistency of ADO release and in vivo efficacy have previously been validated (27). ADO-releasing polymers ( $250 \mathrm{ng} \mathrm{ADO} / \mathrm{d}$ ) or control polymers ( $0 \mathrm{ng} \mathrm{ADO} / \mathrm{d}$ ) were implanted bilaterally into the lateral brain ventricles using a stereotactic implantation device as described (44).

Pharmacology. Bilateral i.c.v. bolus of saline, ADO (5 $\mu \mathrm{g} /$ ventricle), HCY $(250 \mu \mathrm{g} / \mathrm{ventricle})$, or SAM (16 $\mu \mathrm{g} / \mathrm{ventricle})$ was injected using a transiently placed guide cannula and injection volumes of $5 \mu \mathrm{l}$. ITU $(3.1 \mathrm{mg} / \mathrm{kg}$ in $20 \%$ DMSO, i.p.) was administered daily for 5 days to WT and homozygous A1R-knockout mice. Caffeine ( $25 \mathrm{mg} / \mathrm{kg}$ in saline, i.p.) was administered 15 minutes prior to ITU.

Immunohistochemistry. Staining for $\mathrm{ADK}$ and $5 \mathrm{mC}$ was performed using standard protocols (45). For each staining paradigm, high-resolution digital images were acquired under identical conditions and all image processing was applied identically across experimental groups. ADK expression was quantified by densitometry by analyzing fields of $500 \mu \mathrm{m}$ encompassing the entire CA1. Corresponding fields from 2 sections from each animal ( $n=3$ animals per group) were analyzed using ImageJ. Levels in each analysis field were measured as arbitrary density units and are represented as averages \pm SEM normalized to controls. Timm staining was performed and quantified as described (46).

Cell culture. BHK-WT and BHK-AK2 cells (26) were cultured in DMEM (supplemented with $10 \%$ FBS). A pc-DNA3.1 vector with a human CMV promoter was used to drive expression of the ADK-long (provided by R. Gupta, McMasters University, Hamilton, Ontario, Canada) or ADK-short (47) cDNA. Transfection was carried out in parallel experimental replicates using a standard calcium phosphate transfection protocol. Subsequently, cells were harvested for DNA from 3 separate experimental replicates or, to quantify ADK protein expression, 3 transfection replicates were pooled and used for Western blotting.

Western blotting. Cells were harvested for aqueous protein extraction. $25 \mu \mathrm{g}$ of protein was loaded into a $10 \%$ Bis-Tris gel, separated by standard gel electrophoresis, transferred, and incubated overnight using a primary ADK antibody (1:5000) (29). To normalize ADK immunoreactivity with protein loading, a mouse monoclonal anti- $\alpha$-tubulin antibody (sc-8035, 1:5000; Santa Cruz Biotechnology Inc.) was used to reprobe the same blot and the optical density ratio of ADK to $\alpha$-tubulin was calculated.

Global DNA methylation assay. Total genomic DNA was isolated from fresh frozen tissues using a DNeasy Blood and Tissue Kit (QIAGEN). Global DNA methylation status was assessed using the MethylFlash Methylated DNA quantification kit (Epigentek) per the manufacturer's instructions.

DNMT activity assay. Nuclear proteins were isolated from fresh frozen tissue using an EpiQuik Nuclear Extraction Kit I per the manufacturer's instruction (Epigentek). DNMT activity was quantified from freshly isolated nuclear proteins using a fluorimetric EpiQuick DNMT Activity/ Inhibition Assay Ultra kit per the manufacturer's instruction (Epigentek).

MeDIP array. Hippocampal DNA extracts from naive control rats or those sacrificed at $\mathrm{KA}^{9 \mathrm{wk}} / \mathrm{ADO}^{5 \mathrm{~d}}$ (250 $\mathrm{ng}$ ADO per day) were pooled ( $n=3-5$ /experimental group) and analyzed by MeDIP array (05924545001, NimbleGen). The Rat DNA Methylation 3x720K CpG Island Plus RefSeq Promoter array is a rat-specific, whole-genome, tiled format containing at least 3 internal replicates for each probe, with each probe containing from 1 to $18 \mathrm{CpG}$ sites. Tissue samples were prepared according to the manufacturer's instructions, then sent to NimbleGen for hybridization and detection. Each tissue sample was split into 2 fractions, the first enriched for methylated DNA using immunoprecipitation with an antibody against $5 \mathrm{mC}$ and labeled with Cy5 and the second total DNA fraction labeled with Cy 3 . The 2 fractions were mixed and then hybridized on the array. Each probe is represented as the SLR, where the ratio indicates the methylated versus the unmethylated signal. Each ratio is $\log _{2}$ transformed, then scaled using the biweight mean. Gene targets were predicted by associating enriched regions of DNA methylation with their nearest neighbor TSS (upstream or downstream according to the \pm strand) according to the rat RGDS 3.4 assembly. Array data have been deposited in GEO (GSE45932).

Bisulfite sequencing. Hippocampal DNA extracts $(1 \mu \mathrm{g})$ from $\mathrm{KA}^{9 \mathrm{wk}}$ and $\mathrm{KA}^{9 \mathrm{wk}} / \mathrm{ADO}^{5 \mathrm{~d}}$ rat $(n=3 /$ group) were bisulfite converted using the Epitect Bisulfite Conversion Kit according to the manufacturer's protocol (QIAGEN). Primers specific to bisulfite-converted DNA (bs-DNA) were designed using MethPrimer (ref. 48 and Table 1). For the analysis of PolD1, we chose 3 different probes spanning a dSLR range from -3.55 to -2.35 , with each of those probes containing one CPG site. For the analysis of Bat 3 , we chose 2 different probes spanning a dSLR range from -1.52 to -0.92 , with each of those probes containing $1 \mathrm{CpG}$ site. Bisulfite-converted DNA was amplified using HotStarTaq DNA polymerase (QIAGEN) and a standard PCR protocol: initial activation $\left(95^{\circ} \mathrm{C}, 15\right.$ minutes); 40 cycles of denaturation $\left(94^{\circ} \mathrm{C}, 30\right.$ seconds), annealing ( $\mathrm{Tm}$ in Table 1,35 seconds), extension $\left(72^{\circ} \mathrm{C}, 1\right.$ minute); and final extension $\left(72^{\circ} \mathrm{C}, 10\right.$ minutes). Amplified products were gel extracted and ligated into pGEMT Easy Vector and transformed into JM109 competent cells (Promega). Individual clones from each transformation were PCR screened with M13f/r primers for correct insert size. For each DNA target (PolD1 and Bat3), 4-5 clones from each animal were purified and sequenced with M13 primers resulting in 12-15 individual replicates per treatment group (Molecular and Cell Biology Core, Oregon National Primate Research Center). Data were analyzed by aligning sequences with SeqMan Pro Lasergene 10 software (DNAStar) to a theoretical target backbone that is bisulfite converted with methylated CpGs (Bisulfite Sequence Converter, Nephew Lab, Indiana University; http://cancer.informatics.indiana.edu/Nephew_lab/bisulfite_sequencing. $\mathrm{htm})$. Within each sequence, individual $\mathrm{CPG}$ sites were scored as either a cytosine or thymine residue. The percentage methylation was calculated for those probes that cover only $1 \mathrm{CpG}$ site and is presented as pie charts.

Statistics. Quantitative data were analyzed using GraphPAD Prism software. In vivo seizure data from the i.p. KA-injected model are based on $n=8$ 
for $\mathrm{ADO}$ and $n=85$ to 8 for controls depending on dropouts (weeks 18-21 after KA administration) due to lethal seizures in the control groups. Stages 4 and 5 seizure counts were averaged by experimental group as seizures per week and analyzed in 1-week bins. All data are presented as mean \pm SEM. KA seizure data were analyzed using 2-way ANOVA on ranks using an unweighted means analysis followed by a Bonferroni test. Global methylation, IHC, PTZ, and kindling data were analyzed using nonparametric Mann-Whitney 2-tailed $t$ tests assuming non-Gaussian distribution of the data or ANOVAs as appropriate. $P<0.05$ was considered significant.

Study approval. All animal procedures were conducted in a facility accredited by the Association for Assessment and Accreditation of Laboratory Animal Care in accordance with protocols approved by the Legacy Institutional Animal Care and Use Committee.

1. Löscher W, Brandt C. Prevention or modification of epileptogenesis after brain insults: experimental approaches and translational research. Pharmacol Rev. 2010;62(4):668-700.

2. Kobow K, Blümcke I. The methylation hypothesis: do epigenetic chromatin modifications play a role in epileptogenesis? Epilepsia. 2011;52(suppl 4):15-19.

3. Qureshi IA, Mehler MF. Epigenetic mechanisms underlying human epileptic disorders and the process of epileptogenesis. Neurobiol Dis. 2010; 39(1):53-60.

4. Feng J, et al. Dnmt1 and Dnmt3a maintain DNA methylation and regulate synaptic function in adult forebrain neurons. Nat Neurosci. 2010; 13(4):423-430.

5. Ma DK, et al. Neuronal activity-induced Gadd45b promotes epigenetic DNA demethylation and adult neurogenesis. Science. 2009;323(5917):1074-1077.

6. Nelson ED, Kavalali ET, Monteggia LM. Activitydependent suppression of miniature neurotransmission through the regulation of DNA methylation. J Neurosci. 2008;28(2):395-406.

7. Kobow K, et al. Increased reelin promoter methylation is associated with granule cell dispersion in human temporal lobe epilepsy. J Neuropathol Exp Neurol. 2009;68(4):356-364.

8. Lubin FD. Epileptogenesis: can the science of epigenetics give us answers? Epilepsy Curr. 2012; 12(3):105-110

9. Kredich NM, Martin DV. Role of S-adenosylhomocysteine in adenosinemediated toxicity in cultured mouse T lymphoma cells. Cell. 1977;12(4):931-938.

10. Boison D, et al. Neonatal hepatic steatosis by disruption of the adenosine kinase gene. Proc Natl Acad Sci U S A. 2002;99(10):6985-6990.

11. Fredholm BB. Rethinking the purinergic neuronglia connection. Proc Natl Acad Sci U S A. 2012; 109(16):5913-5914.

12. Boison D. Adenosine kinase: exploitation for therapeutic gain. Pharmacol Rev. 2013;65(3):906-943.

13. Pignataro G, Maysami S, Studer FE, Wilz A, Simon RP, Boison D. Downregulation of hippocampal adenosine kinase after focal ischemia as potential endogenous neuroprotective mechanism. J Cereb Blood Flow Metab. 2008;28(1):17-23.

14. James SJ, Melnyk S, Pogribna M, Pogribny IP, Caudill MA. Elevation in S-adenosylhomocysteine and DNA hypomethylation: potential epigenetic mechanism for homocysteine-related pathology. J Nutr. 2002;132(8 suppl):2361S-2366S.

15. Boison D. Adenosine kinase, epilepsy and stroke: mechanisms and therapies. Trends Pharmacol Sci. 2006;27(12):652-658

16. Johansson B, et al. Hyperalgesia, anxiety, and decreased hypoxic neuroprotection in mice lacking the adenosine A1 receptor. Proc Natl Acad Sci US A. 2001;98(16):9407-9412.

\section{Acknowledgments}

This work was supported by the NIH through grants R01NS061844 and P41 EB002520 and by contract W81XWH12-1-0283 from the US Department of Defense. R.L. WilliamsKarnesky is supported by NINDS grant F30NS070359.

Received for publication July 5, 2012, and accepted in revised form May 23, 2013.

Address correspondence to: Detlev Boison, RS Dow Neurobiology Laboratories, Legacy Research Institute, 1225 NE 2nd Ave., Portland, Oregon 97232, USA. Phone: 503.413.1754; Fax: 503.413.5465; E-mail: dboison@downeurobiology.org.
17. Boison D. Adenosine as a modulator of brain activity. Drug News Persp. 2007;20(10):607-611.

18. Lovatt $\mathrm{D}$, et al. Neuronal adenosine release, and not astrocytic ATP release, mediates feedback inhibition of excitatory activity. Proc Natl Acad Sci U S A. 2012; 109(16):6265-6270

19. Boison D, Chen JF, Fredholm BB. Adenosine signalling and function in glial cells. Cell Death Differ. 2010; 17(7):1071-1082.

20. Boison D. Adenosine augmentation therapies (AATs) for epilepsy: prospect of cell and gene therapies. Epilepsy Res. 2009;85(2-3):131-141.

21. $\mathrm{Li} \mathrm{T}$, et al. Adenosine kinase is a target for the prediction and prevention of epileptogenesis in mice. JClin Invest. 2008;118(2):571-582.

22. Masino SA, et al. A ketogenic diet suppresses seizures in mice through adenosine A1 receptors. J Clin Invest. 2011;121(7):2679-2683.

23. Shen HY, Lusardi TA, Williams-Karnesky RL, Lan JQ, Poulsen DJ, Boison D. Adenosine kinase determines the degree of brain injury after ischemic stroke in mice. JCereb Blood Flow Metab. 2011;31(7):1648-1659.

24. Gouder N, Fritschy JM, Boison D. Seizure suppression by adenosine $A_{1}$ receptor activation in a mouse model of pharmacoresistant epilepsy. Epilepsia. 2003;44(7):877-885.

25. Cui XA, Singh B, Park J, Gupta RS. Subcellular localization of adenosine kinase in mammalian cells: The long isoform of AdK is localized in the nucleus. Biochem Biophys Res Commun. 2009; 388(1):46-50

26. Huber A, Padrun V, Deglon N, Aebischer P, Mohler $\mathrm{H}$, Boison D. Grafts of adenosine-releasing cells suppress seizures in kindling epilepsy. Proc Natl Acad Sci U S A. 2001;98(1):7611-7616.

27. Szybala C, Pritchard EM, Wilz A, Kaplan DL, Boison D. Antiepileptic effects of silk-polymer based adenosine release in kindled rats. Exp Neurol. 2009; 219(1):126-135.

28. Wilz A, Pritchard EM, Li T, Lan JQ, Kaplan DL, Boison D. Silk polymer-based adenosine release: Therapeutic potential for epilepsy. Biomaterials. 2008; 29(26):3609-3616.

29. Gouder N, Scheurer L, Fritschy J-M, Boison D. Overexpression of adenosine kinase in epileptic hippocampus contributes to epileptogenesis. J Neurosci. 2004;24(3):692-701.

30. Pitkanen A, Lukasiuk K. Mechanisms of epileptogenesis and potential treatment targets. Lancet Neurol. 2011;10(2):173-186.

31. Dudek FE, Staley KJ. The time course of acquired epilepsy: implications for therapeutic intervention to suppress epileptogenesis. Neurosci Lett. 2011; 497(3):240-246.

32. Dudek FE, Sutula TP. Epileptogenesis in the dentate gyrus: a critical perspective. Prog Brain Res. 2007; 163:755-773.
33. Csoka AB, Szyf M. Epigenetic side-effects of common pharmaceuticals: a potential new field in medicine and pharmacology. Med Hypotheses. 2009; 73(5):770-780.

34. Tsai HC, et al. Transient low doses of DNAdemethylating agents exert durable antitumor effects on hematological and epithelial tumor cells. Cancer Cell. 2012;21(3):430-446.

35. Zampieri M, et al. ADP-ribose polymers localized on Ctcf-Parp1-Dnmt1 complex prevent methylation of Ctcf target sites. Biochem J. 2012;441(2):645-652.

36. Henshall DC, Sinclair J, Simon RP. Relationship between seizure-induced transcription of the DNA damage-inducible gene GADD45, DNA fragmentation, and neuronal death in focally evoked limbic epilepsy. J Neurochem. 1999;73(4):1573-1583.

37. Aronica E, et al. Upregulation of adenosine kinase in astrocytes in experimental and human temporal lobe epilepsy. Epilepsia. 2011;52(9):1645-1655.

38. Rebola N, et al. Decrease of adenosine $A_{1}$ receptor density and of adenosine neuromodulation in the hippocampus of kindled rats. EurJ Neurosci. 2003; 18(4):820-828.

39. Boison D. The adenosine kinase hypothesis of epileptogenesis. Prog Neurobiol. 2008;84(3):249-262.

40. Gray JH, Owen RP, Giacomini KM. The concentrative nucleoside transporter family, SLC28. Pflugers Arch. 2004;447(5):728-734.

41. Baldwin SA, Beal PR, Yao SY, King AE, Cass CE, Young JD. The equilibrative nucleoside transporter family, SLC29. Pflugers Arch. 2004;447(5):735-743.

42. de Tisi J, et al. The long-term outcome of adult epilepsy surgery, patterns of seizure remission, and relapse: a cohort study. Lancet. 2011; 378(9800):1388-1395

43. White HS, Wolf HH, Woodhead JH, Kupferberg HJ. The National Institutes of Health Anticonvulsant Drug Development Program: screening for efficacy. Adv Neurol. 1998;76:29-39.

44. Boison D, Huber A, Padrun V, Deglon N, Aebischer $\mathrm{P}$, Mohler H. Seizure suppression by adenosinereleasing cells is independent of seizure frequency. Epilepsia. 2002;43(8):788-796.

45. Studer FE, et al. Shift of adenosine kinase expression from neurons to astrocytes during postnatal development suggests dual functionality of the enzyme. Neuroscience. 2006;142(1):125-137.

46. Cavazos JE, Golarai G, Sutula TP. Mossy fiber synaptic reorganization induced by kindling: time course of development, progression, and permanence. J Neurosci. 1991;11(9):2795-2803.

47. Fedele DE, et al. Astrogliosis in epilepsy leads to overexpression of adenosine kinase resulting in seizure aggravation. Brain. 2005;128(Pt 10):2383-2395.

48. Li LC, Dahiya R. MethPrimer: designing primers for methylation PCRs. Bioinformatics. 2002; 18(11):1427-1431. 